\title{
The prevalence of human papillomavirus infections and associated risk factors in men-who-have-sex-with-men in Cape Town, South Africa
}

Etienne E. Müller ${ }^{1 *}$, Kevin Rebe $2,3,4$, Tobias F. Chirwa ${ }^{5}$, Helen Struthers ${ }^{2,3,4}$, James Mclntyre ${ }^{2,3,6}$ and David A. Lewis ${ }^{7,8}$

\begin{abstract}
Background: We investigated the prevalence of human papillomavirus (HPV) infection and associated behavioural risk factors in men-who-have-sex-with-men (MSM) attending a clinical service in Cape Town, South Africa.

Methods: MSM were enrolled at the Ivan Toms Centre for Men's Health in Cape Town. A psychosocial and sexual behavioral risk questionnaire was completed for each participant and urine, oro-pharyngeal and anal swabs were collected for HPV testing using the Linear Array HPV Genotyping Test. Logistic regression analyses were performed to determine sexual risk factors associated with HPV infection at the three anatomical sites.
\end{abstract}

Results: The median age of all 200 participants was 32 years (IQR 26-39.5), of which $31.0 \%$ were black, $31.5 \%$ mixed race/coloured and $35.5 \%$ white. The majority of the participants (73.0 \%) had completed high school, $42.0 \%$ had a tertiary level qualification and $69.0 \%$ were employed. HPV genotypes were detected in $72.8 \%$ [95 \% Cl: 65. 9-79.0 \%], $11.5 \%$ [95 \% Cl: 7.4-16.8 \%] and $15.3 \%$ [95 \% Cl: 10.5-21.2\%] of anal, oro-pharyngeal and urine specimens, respectively. Prevalence of high-risk (HR)-HPV types was $57.6 \%$ [95 \% Cl: 50.3-64.7\%] in anal samples, $7.5 \%$ [95 \% Cl: 4.3-12.1 \%] in oro-pharyngeal samples and 7.9 \% [95 \% Cl: 4.5-12.7\%] in urine, with HPV-16 being the most common HR-HPV type detected at all sites. HPV-6/11/16/18 was detected in $40.3 \%$ [95 \% Cl: 33.3-47.6 \%], $4.5 \%$ [95 \% Cl: 2.1-8.4 \%] and 3.2 \% [95 \% Cl: 1.2-6.8 \%] of anal, oro-pharyngeal and urine samples, respectively. Multiple HPV types were more common in the anal canal of MSM while single HPV types constituted the majority of HPV infections in the oropharynx and urine. Among the 88 MSM (44.0\%) that were HIV positive, $91.8 \%$ [95 \% Cl: 83.8-96.6 \%] had an anal HPV infection, 81.2 \% [95 \% Cl: 71.2-88.8 \%] had anal HR-HPV and $85.9 \%$ [95 \% Cl: $76.6-92.5 \%$ had multiple anal HPV types. Having sex with men only, engaging in group sex in lifetime, living with HIV and practising receptive anal intercourse were the only factors independently associated with having any anal HPV infection.

Conclusions: Anal HPV infections were common among MSM in Cape Town with the highest HPV burden among HIV co-infected MSM, men who have sex with men only and those that practiced receptive anal intercourse. Behavioural intervention strategies and the possible roll-out of HPV vaccines among all boys are urgently needed to address the high prevalence of HPV and HIV co-infections among MSM in South Africa.

Keywords: HPV, Anal, Oro-pharyngeal, Urine, MSM, Risk factors, South Africa

\footnotetext{
* Correspondence: etiennem@nicd.ac.za

${ }^{1}$ Centre for HIV and Sexually Transmitted Infections, National Institute for Communicable Diseases, National Health Laboratory Service, Johannesburg, South Africa

Full list of author information is available at the end of the article
} 


\section{Background}

Human papillomavirus (HPV) is the most common sexually transmitted viral infection worldwide and is associated with occurrence of warts (condylomas) and a variety of cancers in both men and women [1]. The outcome of HPV infection depends on the specific HPV type/s present and can range from asymptomatic infection to severe squamous cell malignancies. Low-risk HPV types (such as types 6 and 11) are associated with anogenital warts and mild dysplasia, while high-risk types (such as 16 and 18) are associated with high-grade dysplasia and cancers of the cervix, vulva, vagina, urethra, penis, anus and oropharynx [1]. HPV is an independent risk factor for the acquisition of HIV [2]. HIV infection is also associated with an increased risk of acquiring new HPV infections and a reduction in the rate of HPV clearance [3]. South Africa has one of the highest HIV prevalence rates globally with an estimated prevalence of $12.2 \%$ in the general population in 2012 [4]. HIV is especially prevalent among men-who-havesex-with-men (MSM), with estimated prevalence rates of $22.3 \%, 26.8 \%$ and $48.2 \%$ in Cape Town, Johannesburg and Durban, respectively [5]. Unprotected receptive anal intercourse is up to sixteen times more likely to transmit HIV than unprotected vaginal sex, placing MSM at higher risk of acquiring and transmitting HIV [6]. A meta-analysis of 53 studies reported HPV co-infection in $89 \%-93 \%$ of HIV-infected MSM [7].

HPV infection in men is an important concern due to its association with anal, urethral, penile and oropharyngeal cancers. In South Africa, relatively little is known about the incidence rates of these cancers in men, and although these cancers are less common than cervical cancer, their association with HPV infection make them amenable to similar preventative measures as those for cervical cancer. The risk of developing invasive anal cancer was 17 times higher among MSM than in men-who-have-sex-with-women (MSW) and the risk doubled in HIV-infected MSM [8]. A number of studies reported anal HPV prevalence rates of 30-70\% in HIV-negative MSM and 71-100 \% in HIV-positive MSM [9-12]. Oral HPV infection is less common in adults than anogenital HPV infection but is still strongly associated with HIV infection, high-risk sexual practices, especially engaging in oro-genital sex and having a high number of sexual partners $[13,14]$. HPV can also be found in the urinary tract, indicating HPV infection in either the distal or proximal urethra, prostate and/or urinary bladder [15]. There are currently three HPV vaccines available that were designed to prevent HPV-associated cancer to certain HR-HPV genotypes. These HPV vaccines include the bivalent Cervarix ${ }^{\circledast}$ HPV vaccine (GSK Aspen) (targeting HPV types 16 and 18), the quadrivalent Gardasil $^{\bullet} \mathrm{HPV}$ vaccine (MSD) (targeting HPV types $6,11,16$ and 18) as well as the 9-valent Gardasil ${ }^{\circ}$ $9 \mathrm{HPV}$ vaccine (MSD) (targeting HPV types 6, 11, 16, 18, 31, 33, 45, 52 and 58).

Epidemiological research has remained very limited among the MSM population in South Africa and as a result there are currently no HPV prevalence data for MSM. Due to high HIV prevalence rates among MSM in South Africa, this group has been identified by the South African Department of Health for targeted healthcare interventions and HIV prevention programs [16]. This study will therefore aim to investigate the prevalence of HPV genotypes at different anatomical sites (anus, oropharynx and urine) in a sample of 200 MSM seeking care at a dedicated men's clinic in Cape Town, South Africa.

\section{Methods}

\section{Population and sample selection}

This study is a secondary analysis of data collected during a cross-sectional study of symptomatic and asymptomatic STIs among MSM in Cape Town, South Africa [17]. A convenience sample of 200 sequential MSM were enrolled by Health4Men (Anova Health Institute) at the Ivan Toms Centre for Men's Health (ITCMH) in Cape Town during 2011 and 2012. These MSM were mostly asymptomatic $(71.0 \%)$ for STIs and attended the ITCMH for HIV, sexual health and/or mental health care and for commodities such as condoms and lubricants. The inclusion criteria for study participation included being 18 years of age or older, having sex with other men within one year prior to recruitment, understanding the study procedures, risks and benefits and willingness to give informed consent. Study participants attended two study-related visits two weeks apart. During the first visit participants were assessed for STI symptoms and examined for STI and HIV-related clinical conditions. All participants completed a researcher administered study questionnaire and provided a selfcollected urine specimen and clinician collected rectal and oro-pharyngeal swab. These specimens were tested for Neisseria gonorrhoeae and Chlamydia trachomatis using the RNA-based Aptima Combo 2 assay (HologicGen-Probe, San Diego, CA, USA.) and stored at $-70{ }^{\circ} \mathrm{C}$ for HPV testing. At the follow-up visit, results were relayed to all participants and diagnosed conditions were treated according to the South African Department of Health's first-line comprehensive management and control of STIs protocol [18]. All 200 participants gave permission for their specimens to be used for future research. Ethical approvals for this study were obtained from the Human Research Ethics Committee (Medical) of the University of the Witwatersrand [protocol M120454] and the Bioethics Department at the University of Cape Town [protocol 419/2011]. 


\section{Psychosocial and sexual behavioural data collection}

Socio-demographic and sexual behavior characteristics were collected by designated study staff for the primary study through personal interviews using a standardized questionnaire. Circumcision status was confirmed during clinical examination. A sexual partner matrix (SPM) for the last 10 sexual encounters in the past 12 months (or less if the participant had less than 10 sexual encounters in the past 12 months) was completed for each participant. The SPM recorded the gender of each partner and condom use during sexual practices that included receptive and insertive anal intercourse, oral sex and oro-anal sex. Vaginal intercourse was recorded for men who had sexual encounters with female partners in the past 12 months.

\section{HPV detection and genotyping}

HPV testing was performed at the STI Section, Centre for HIV and STIs, National Institute for Communicable Diseases (NICD), in Johannesburg. Three archived specimens for each of the 200 enrolled MSM participants were analysed, which included a first-void urine specimen, an oro-pharyngeal swab and an anal swab, which were all previously stored at $-70{ }^{\circ} \mathrm{C}$ at the STI Section, Centre for HIV and STIs, NICD.

Genomic DNA was extracted from urine and swab specimens using the MagNAPure Compact Automated DNA Extractor (Roche) and used for HPV genotyping. The Linear Array (LA) HPV Genotyping Test (Roche Molecular Systems, Inc., Branchburg, NJ, USA) was used to determine the HPV genotype distribution in these 600 specimens. The LA test amplified the target HPV DNA for 37 anogenital HPV genotypes, which included 24 low risk HPV types $(6,11,26,40,42,53,54,55,61,62,64$, $66,67,69,70,71,72,73,81,82,83,84$, IS39 and CP6108) and 13 high risk HPV types $(16,18,31,33,35$, $39,45,51,52,56,58,59$ and 68$)$. HPV-52 was only recorded positive in the absence of HPV types 33, 35 and 58 due to the combined probe used for these 4 types in the LA assay. The $\beta$-globin gene was amplified as a control for cell adequacy, extraction and amplification. Samples with a negative $\beta$-globin result and a positive HPV DNA result were considered valid and adequate for analyses.

\section{Detection of HIV and other STIs}

Laboratory detection of HIV, syphilis, gonorrhea and chlamydia infection at the urethral, anal and oro-pharyngeal sites were previously determined for the primary study [17]. Syphilis testing was done on site using the SD Bioline rapid syphilis test and HIV serostatus was determined using both the SD Bioline HIV 1/2 3.0 test (Standard Diagnostics, Korea) and the Alere Determine HIV-1/2 kit (Alere Medical, Japan). Specimens were previously analysed for gonorrheal and chlamydial infection at the
STI Section, NICD, using the Aptima 2 Combo assay (Hologic-Gen-Probe, San Diego, CA, USA.).

\section{Data confidentiality}

Individual specimens and questionnaire data were delinked from all personal identifiers and each participant was assigned with a unique random HPV study number to ensure anonymity. There were no documents in existence linking original study numbers with HPV study numbers. The HPV genotyping results obtained were therefore not relayed to any patients and the results were only used for research purposes.

\section{Variables and definitions}

The main outcome variables consisted of type-specific HPV prevalence, any HPV type, HR-HPV types and multiple HPV types. A participant was considered to have a HR-HPV infection if he tested positive for one or more HR- HPV genotypes, irrespective of the participant being co-infected with one or more LR-HPV genotypes. Multiple HPV was defined in participants with two or more HR or LR HPV genotypes. Socio-demographic variables of interest included age, gender, ethnicity, dwelling location, level of education, job status and income. For the purpose of this study MSM consisted of MSM not having sex with women (MSM only) and men who had sex with both men and women (MSMW). Additional sexual behaviours included last sex with men and women, number of male and female partners in the past 12 months, having a main partner, sex at sex-on-site venues in past 12 months, sex with a partner met on the internet in past 12 months, engaging in group sex in past 12 months and lifetime, sharing sex toys, participating in transactional sex, sex while under the influence of alcohol and drugs, lubrication used during sex and having an STI diagnosed in the past 12 months.

\section{Statistical analyses}

HPV prevalence and questionnaire data were entered and analysed using both Epi Info version 7 (USDHHS, Centers for Disease Control and Prevention) and STATA SE version 14.0 (Stata Corporation, College Station, Texas, USA). Descriptive statistics included frequency tables for categorical variables such as level of education, HR-HPV and multiple HPV and summary measures i.e. mean and standard deviation (SD) or median and interquartile range (IQR) for continuous variables such as age. Factors associated with HPV infection, comparing differences between MSM only and MSMW and differences between HIV-positive and HIV-negative participants were assessed with Pearson chi-square test and Fisher's exact test for categorical variables and Student's $t$-test for continuous variables. Factors with a $p$-value of $\leq 0.05$ in the univariate logistic regression 
analyses were included in the multiple logistic regression model to determine possible associations with outcome variables. The HPV outcome variables included HPV prevalence rates in this selected study population for any HPV, HR-HPV and multiple HPV at the 3 anatomical sites and their $95 \%$ confidence intervals (CI); crude odds ratios (COR) and adjusted odds ratios (AOR) and its $95 \% \mathrm{CI}$ were calculated.

\section{Results}

\section{Demographic and behavioural characteristics}

Baseline characteristics, sexual behaviour and STI risks for the 200 participants have been published [17]. Briefly, the median age of all 200 participants was 32 years (IQR 26-39.5) with three-quarters (150) under 40 years of age. A total of fifteen participants (7.5\%) identified as transgender women (TGW; male to female). All three major South African racial groups were equally represented with $62(31.0 \%)$ black, 63 (31.5\%) mixed race/coloured and 71 (35.5\%) white participants. Baseline and sexual behavioural characteristics of men who have sex with men only (MSM only) and men who have sex with men and women (MSMW) are summarised in Table 1.

The majority of MSM participants $(155 ; 77.5 \%)$ indicated that they had sex with men only while 45 (22.5\%) had sex with both men and women in the preceding 12 months. More than a third of all participants (77; $38.5 \%$ ), reported transactional sex (paid and/or received money for sex) in the past year. Significantly more MSMW reported participating in transactional sex in the past year $(p<0.001)$ and having had sex while under the influence of drugs in their lifetime $(p=0.004)$. More than half $(52.3 \%)$ of all MSM only participants were HIV positive while only $15.6 \%$ of MSMW had an HIV infection $(p<0.001)$.

Insertive and receptive anal sex in the last 12 months were reported by $143(71.5 \%)$ and 140 (70.0 \%) participants, respectively. Among MSMW, insertive anal sex was more commonly practised than receptive anal sex (86.5 \% vs $33.3 \%$ ), while MSM only reported more receptive anal sex than insertive anal sex $(80.6 \%$ vs $67.1 \%)$. Compared to MSM only, significantly more MSMW practised insertive anal sex $(p=0.010)$ and less receptive anal sex $(p<0.001)$. MSM only participants practised significantly more receptive oral sex $(95.5 \%$ vs $57.8 \%$ ) and insertive oro-anal sex (43.2\% vs $22.2 \%$ ) in the last 12 months. The SPM analysis revealed that 36 (80.0 \%) MSMW participants had a female partner among their last 10 sexual partners. Performing vaginal intercourse was reported by 34 (94.4\%) of these MSMW participants, performing anal sex on women was less common $(15 / 36 ; 41.7 \%)$, while performing and receiving oral sex with women was reported by 29 (80.6 \%) and $22(61.1 \%)$ participants, respectively. Performing and receiving oro-anal sex with women was only reported by $7 / 36(19.4 \%)$ participants.

\section{HIV and STI prevalence}

As previously reported, 47 (23.5\%) participants reported having an STI diagnosis within the previous year, mostly with a single STI episode (80.9\% of cases) [17]. Eighty eight (44.0\%) participants were HIV positive with the majority (74.1 \%) having CD4 counts below 500 cells/ $\mu \mathrm{l}$. Twenty two (11.0 \%) participants tested positive for syphilis antibodies. Neisseria gonorrhoeae and Chlamydia trachomatis infections were detected in 32 (16\%) and 23 (11.5\%) MSM, respectively.

\section{HPV prevalence and genotype distribution}

As shown in Tables 2 and 3, a total of 191 anal swabs (95.5\%), 200 oro-pharyngeal swabs (100\%) and 190 urines (95\%) had a valid HPV DNA result and were ncluded in the analysis; test strips lacking human beta globin DNA detection were reported as invalid. An overall HPV prevalence of 75 \% (150/200) [95 \% CI: 68.4-80.8 \%] was observed for all patients, irrespective of the site of HPV positivity. HPV genotypes were detected in 139/191 (72.8 \%) [95 \% CI: 65.9-79.0\%], 23/200 (11.5 \%) [95 \% CI: 7.4-16.8 \%] and 29/190 (15.3\%) [95 \% CI: 10.5-21.2\%] of anal, oro-pharyngeal and urine specimens, respectively. The mean number of HPV types observed in anal, oropharyngeal and urine specimens were 4.47, 1.74 and 1.41 $\mathrm{HPV}$ types in HPV-positive MSM, respectively. Multiple HPV types were observed in 113/191 (59.2\%) [95 \% CI: 51.8-66.2 \%] of anal specimens, 7/200 (3.5\%) [95\% CI: 1.4-7.1\%] of oro-pharyngeal specimens and 7/190 (3.7 \%) [95 \% CI: 1.5-7.4 \%] of urine specimens (Fig. 1). The most HPV types observed in an anal specimen was 15 , followed by $6 \mathrm{HPV}$ types in an oro-pharyngeal specimen and 4 HPV types in a urine specimen.

For anal specimens, a total of 110 (57.6 \%) [95 \% CI: 50.3-64.7\%] were HR-HPV positive. HR-HPV types were less prevalent in the pharynx and urine, with 15 (7.5 \%) [95 \% CI: 4.3-12.1 \%] oro-pharyngeal specimens and 15 (7.9 \%) [95 \% CI: 4.5-12.7\%] urine specimens testing positive for HR-HPV. HPV types 16 and 18, the only HR-HPV types included in both the bivalent and quadrivalent HPV vaccines, were detected in 55 (28.8\%) [95 \% CI: 22.5-35.8 \%] anal specimens, 6 (3.0\%) [95 \% CI: $1.1-6.4 \%$ ] oro-pharyngeal specimens and 3 (1.6\%) [95\% CI: $0.3-4.5 \%$ ] urine specimens. The prevalence rates of all HPV genotypes included in the quadrivalent HPV vaccine (HPV-6/11/16/18) were 77 (40.3\%) [95 \% CI: 33.3$47.6 \%$ ], 9 (4.5\%) [95 \% CI: 2.1-8.4\%] and 6 (3.2\%) [95\% CI: $1.2-6.8 \%$ ] in anal, oro-pharyngeal and urine samples, respectively. HPV types included in the new 9-valent HPV vaccine (HPV-6/11/16/18/31/33/45/52/58) were detected in 110 (57.6 \%) [95 \% CI: 50.3-64.7\%], 12 (6.0\%) [95 \% 
Table 1 Baseline and sexual behavioural characteristics stratified according to men who have sex with men only (MSM only) and men who have sex with men and women (MSMW)

\begin{tabular}{|c|c|c|c|c|}
\hline \multirow[t]{2}{*}{ Variable } & \multicolumn{3}{|l|}{ No. (\%) of men } & \multirow[t]{2}{*}{$p$-value } \\
\hline & MSM only $(n=155)$ & MSMW $(n=45)$ & Totals $(n=200)$ & \\
\hline Age [years(IQR)] & $33(26-40)$ & $28(24-37)$ & $32(26-39.5)$ & 0.025 \\
\hline \multicolumn{5}{|l|}{ Gender } \\
\hline Male & $140(90.3)$ & $45(100.0)$ & $185(92.5)$ & \multirow[t]{2}{*}{0.030} \\
\hline Transgender & $15(9.7)$ & $0(0.0)$ & $15(7.5)$ & \\
\hline \multicolumn{5}{|l|}{ Ethnicity } \\
\hline Black & $48(31.0)$ & $14(31.1)$ & $62(31.0)$ & \multirow[t]{4}{*}{$<0.001$} \\
\hline Coloured & $38(24.5)$ & $25(55.6)$ & $63(31.5)$ & \\
\hline White & $65(41.9)$ & $6(13.3)$ & $71(35.5)$ & \\
\hline Other & $4(2.6)$ & $0(0.0)$ & $4(2.0)$ & \\
\hline \multicolumn{5}{|l|}{ Education } \\
\hline Completed high school & $122(78.7)$ & $33(73.3)$ & $145(72.5)$ & \multirow[t]{2}{*}{$<0.001$} \\
\hline Tertiary qualification & $73(47.1)$ & $11(24.4)$ & $84(42.0)$ & \\
\hline \multicolumn{5}{|l|}{ Transactional sex } \\
\hline No & $112(72.3)$ & $11(24.4)$ & $123(61.5)$ & \multirow[t]{4}{*}{$<0.001$} \\
\hline Yes, paid money for sex & $17(11.0)$ & $7(15.6)$ & $24(12.0)$ & \\
\hline Yes, received money for sex & $21(13.5)$ & $15(33.3)$ & $36(18.0)$ & \\
\hline Yes, paid and received money for sex & $5(3.2)$ & $12(26.7)$ & $17(8.5)$ & \\
\hline \multicolumn{5}{|l|}{ Sex while under the influence of drugs } \\
\hline Yes & $73(47.1)$ & $32(71.1)$ & $105(52.5)$ & \multirow[t]{2}{*}{0.004} \\
\hline No & $82(52.9)$ & $13(28.9)$ & $95(47.5)$ & \\
\hline \multicolumn{5}{|l|}{ HIV serostatus } \\
\hline Positive & $81(52.3)$ & $7(15.6)$ & $88(44.0)$ & \multirow[t]{2}{*}{$<0.001$} \\
\hline Negative & $74(47.7)$ & $38(84.4)$ & $112(56.0)$ & \\
\hline \multicolumn{5}{|l|}{ Frequency of insertive anal sex } \\
\hline Yes & $104(67.1)$ & $39(86.7)$ & $143(71.5)$ & \multirow[t]{2}{*}{0.010} \\
\hline No & $51(32.9)$ & $6(13.3)$ & $57(28.5)$ & \\
\hline \multicolumn{5}{|l|}{ Frequency of receptive anal sex } \\
\hline Yes & $125(80.6)$ & $15(33.3)$ & $140(70.0)$ & \multirow[t]{2}{*}{$<0.001$} \\
\hline No & $30(19.4)$ & $30(66.7)$ & $60(30.0)$ & \\
\hline \multicolumn{5}{|l|}{ Frequency of receptive oral sex } \\
\hline Yes & $148(95.5)$ & $26(57.8)$ & $174(87.0)$ & \multirow[t]{2}{*}{$<0.001$} \\
\hline No & $7(4.5)$ & $19(42.2)$ & $26(13.0)$ & \\
\hline \multicolumn{5}{|l|}{ Performing oro-anal sex (rimming) } \\
\hline Yes & $67(43.2)$ & $10(22.2)$ & $77(38.9)$ & \multirow[t]{2}{*}{0.011} \\
\hline No & $88(56.8)$ & $35(77.8)$ & $123(61.5)$ & \\
\hline
\end{tabular}

CI: $3.1-10.3 \%$ ] and 10 (5.3 \%) [95\%CI: 2.6-9.5\%] anal, oro-pharyngeal and urine specimens, respectively.

The most common HPV types detected in anal specimens were HPV-16 (42; 22 \%), HPV-6 (39; 20.4\%) and HPV-51 (31; $16.2 \%)$. In oro-pharyngeal specimens, the most common HPV types detected were HPV-16 (6; $3.0 \%)$, HPV-72 (4; $2.0 \%$ ), followed by HPV-35 and HPV51 (both $3 ; 1.5 \%$ ). The most prevalent HPV types in urine were HPV-16, HPV-45, HPV-55, HPV-59, HPV-61, HPV62, HPV-66 and HPV-70 (all 3; $1.6 \%$ ). The HPV type specific prevalence rates in anal, oro-pharyngeal and urine specimens are summarised in Fig. 2.

\section{HPV and HIV co-infections}

A total of 88 (44.0\%) participants tested HIV-positive in this study. Among the $85 \mathrm{HIV}$-positive participants with 
Table 2 HPV and HIV-co-infection at 3 anatomical sites among MSM in Cape Town, South Africa

\begin{tabular}{|c|c|c|c|c|c|c|c|c|c|c|c|c|}
\hline \multirow{2}{*}{$\begin{array}{l}\text { HPV type } \\
\text { classification }\end{array}$} & \multicolumn{4}{|c|}{$\operatorname{ANAL}(n=191)$} & \multicolumn{4}{|c|}{ ORO-PHARYNGEAL $(n=200)$} & \multicolumn{4}{|c|}{ URINE $(n=190)$} \\
\hline & Total (\%) & $\begin{array}{l}\text { HIV- } \\
(n=106)\end{array}$ & $\begin{array}{l}\mathrm{HIV}+ \\
(n=85)\end{array}$ & $P$-value & Total (\%) & $\begin{array}{l}\text { HIV- } \\
(n=112)\end{array}$ & $\begin{array}{l}\mathrm{HIV+} \\
(n=88)\end{array}$ & $P$-value & Total (\%) & $\begin{array}{l}\text { HIV- } \\
(n=107)\end{array}$ & $\begin{array}{l}\mathrm{HIV}+ \\
(n=83)\end{array}$ & $P$-value \\
\hline No type & $52(27.2)$ & $45(42.4)$ & $7(8.2)$ & $<0.001$ & $177(88.5)$ & $104(92.9)$ & $73(83.0)$ & 0.029 & $161(84.7)$ & $91(85.1)$ & $70(84.3)$ & 0.892 \\
\hline Any type & $139(72.8)$ & $61(57.6)$ & 78 (91.8) & $<0.001$ & $23(11.5)$ & $8(7.1)$ & $15(17.1)$ & 0.029 & $29(15.3)$ & $16(14.3)$ & $13(14.8)$ & 0.892 \\
\hline Single type & $26(13.6)$ & $21(19.8)$ & $5(5.9)$ & 0.005 & $16(8.0)$ & $6(5.4)$ & $10(11.4)$ & 0.120 & $22(11.6)$ & $14(12.5)$ & $8(9.1)$ & 0.461 \\
\hline $\begin{array}{l}\text { Multiple } \\
\text { types }\end{array}$ & $113(59.2)$ & $40(37.7)$ & $73(85.9)$ & $<0.001$ & $7(3.5)$ & $2(1.8)$ & $5(5.7)$ & 0.136 & $7(3.7)$ & $2(1.8)$ & $5(5.7)$ & 0.131 \\
\hline LR-HPV & $126(66.0)$ & $50(47.2)$ & 76 (89.4) & $<0.001$ & $14(7.0)$ & $4(3.6)$ & $10(11.4)$ & 0.032 & $19(10.0)$ & $8(7.5)$ & $11(13.3)$ & 0.188 \\
\hline HR-HPV & $110(57.6)$ & $41(38.7)$ & 69 (81.2) & $<0.001$ & $15(7.5)$ & $6(5.4)$ & $9(10.2)$ & 0.194 & $15(7.9)$ & $9(8.4)$ & $6(7.2)$ & 0.764 \\
\hline HPV-6/11 & $50(26.2)$ & 19 (17.9) & 31 (36.5) & 0.003 & $3(1.5)$ & $1(0.9)$ & $2(2.3)$ & 0.425 & $3(1.6)$ & $0(0.0)$ & $3(3.6)$ & 0.047 \\
\hline HPV-16/18 & $55(28.8)$ & 19 (17.9) & $36(42.4)$ & $<0.001$ & $6(3.0)$ & $3(2.7)$ & $3(3.4)$ & 0.763 & $3(1.6)$ & $2(1.9)$ & $1(1.2)$ & 0.715 \\
\hline $\begin{array}{l}\text { HPV-6/11 } \\
/ 16 / 18\end{array}$ & 77 (40.3) & $30(28.3)$ & 47 (55.3) & $<0.001$ & $9(4.5)$ & $4(3.6)$ & $5(5.7)$ & 0.474 & $6(3.2)$ & $2(1.9)$ & $4(4.8)$ & 0.248 \\
\hline $\begin{array}{l}\text { HPV-31 } \\
133 / 45\end{array}$ & $50(26.2)$ & $14(13.2)$ & $36(42.4)$ & $<0.001$ & $3(1.5)$ & $0(0.0)$ & $3(3.4)$ & 0.048 & $5(2.6)$ & $3(2.8)$ & $2(2.4)$ & 0.866 \\
\hline $\begin{array}{l}\text { HPV-6/11/16 } \\
\text { /18/31/33/45 }\end{array}$ & $98(51.3)$ & $36(34.0)$ & 62 (72.9) & $<0.001$ & $11(5.5)$ & $4(3.6)$ & $7(8.0)$ & 0.177 & $10(5.3)$ & $5(4.7)$ & $5(6.0)$ & 0.679 \\
\hline
\end{tabular}

valid anal HPV results, 78 (91.8 \%) were co-infected with any anal HPV $(p<0.001), 73(85.9 \%)$ had multiple anal HPV types $(p<0.001), 76$ (89.4 \%) had LR-HPV $(p<0.001)$ and $69(81.2 \%)$ were co-infected with HR-HPV $(p<0.001)$ (Table 2). Quadrivalent HPV vaccine types 6, 11, 16 and 18 were detected in $47(55.3 \%)$ anal specimens of HIV-positive participants $(p<0.001)$, and increased to $62(72.9 \%)$ with the addition of cross-protection HPV types 31,33 and $45(p<0.001)$. Having any oropharyngeal HPV infection $(15 / 88 ; 17.1 \%)$ and having a LR-HPV oro-pharyngeal infection (10/88; $11.4 \%$ ) were significantly associated with being HIV-positive
( $p=0.029$ and $p=0.032$ respectively). Multiple anal HPV types were detected more frequently in HIV-positive MSM (85.9 \% vs 37.7 \% in HIV-negative MSM). The mean number of HPV types detected in HPV-positive anal samples was 5.7 (range 1-15) in HIV-positive participants and 2.9 (range $1-10)$ in HIV-negative participants $(p<0.001)$.

\section{HPV according to sex partner status}

Significant differences in anal HPV prevalence were observed between men who had sex with men only (MSM only) and men who had sex with men and women (MSMW) (Table 3). MSMW were less likely to have any

Table 3 HPV infection among MSM only and MSMW at 3 anatomical sites among MSM in Cape Town, South Africa

\begin{tabular}{|c|c|c|c|c|c|c|c|c|c|c|c|c|}
\hline \multirow{2}{*}{$\begin{array}{l}\text { HPV type } \\
\text { classification }\end{array}$} & \multicolumn{4}{|c|}{$\operatorname{ANAL}(n=191)$} & \multicolumn{4}{|c|}{ ORO-PHARYNGEAL $(n=200)$} & \multicolumn{4}{|c|}{ URINE $(n=190)$} \\
\hline & $\overline{\text { Total }(\%)}$ & $\begin{array}{l}\text { MSM only } \\
(n=150)\end{array}$ & $\begin{array}{l}\text { MSMW } \\
(n=41)\end{array}$ & $P$-value & Total (\%) & $\begin{array}{l}\text { MSM only } \\
(n=155)\end{array}$ & $\begin{array}{l}\text { MSMW } \\
(n=45)\end{array}$ & $P$-value & Total (\%) & $\begin{array}{l}\text { MSM only } \\
(n=145)\end{array}$ & $\begin{array}{l}\text { MSMW } \\
(n=45)\end{array}$ & $P$-value \\
\hline No type & $52(27.2)$ & $22(14.8)$ & $30(73.2)$ & $<0.001$ & $177(88.5)$ & $133(85.8)$ & $44(97.8)$ & 0.027 & $161(84.7)$ & $123(84.8)$ & $38(84.4)$ & 0.950 \\
\hline Any type & $139(72.8)$ & $128(85.3)$ & $11(26.8)$ & $<0.001$ & $23(11.5)$ & $22(14.2)$ & $1(2.2)$ & 0.027 & $29(15.3)$ & $22(15.2)$ & $7(15.6)$ & 0.950 \\
\hline Single type & $26(13.6)$ & $22(14.7)$ & $4(9.8)$ & 0.416 & $16(8.0)$ & $15(9.7)$ & $1(2.2)$ & 0.105 & $22(11.6)$ & $18(12.4)$ & $4(8.9)$ & 0.519 \\
\hline $\begin{array}{l}\text { Multiple } \\
\text { types }\end{array}$ & $113(59.2)$ & $106(70.7)$ & $7(17.1)$ & $<0.001$ & $7(3.5)$ & $7(4.5)$ & $0(0.0)$ & 0.147 & $7(3.7)$ & $4(2.8)$ & $3(6.7)$ & 0.224 \\
\hline LR-HPV & $126(66.0)$ & $117(78.0)$ & $9(22.0)$ & $<0.001$ & $14(7.0)$ & $13(8.4)$ & $1(2.2)$ & 0.154 & $19(10.0)$ & $15(10.3)$ & $4(8.9)$ & 0.776 \\
\hline HR-HPV & $110(57.6)$ & $102(68.0)$ & $8(19.50$ & $<0.001$ & $15(7.5)$ & $15(9.7)$ & $0(0.0)$ & 0.030 & $15(7.9)$ & $10(6.9)$ & $5(11.1)$ & 0.359 \\
\hline HPV-6/11 & $50(26.2)$ & $48(32.0)$ & $2(4.9)$ & $<0.001$ & $3(1.5)$ & $3(1.9)$ & $0(0.0)$ & 0.347 & $3(1.6)$ & $3(2.1)$ & $0(0.0)$ & 0.331 \\
\hline HPV-16/18 & $55(28.8)$ & $52(34.7)$ & $3(7.3)$ & $<0.001$ & $6(3.0)$ & $6(3.9)$ & $0(0.0)$ & 0.180 & $3(1.6)$ & $2(1.4)$ & $1(2.2)$ & 0.692 \\
\hline $\begin{array}{l}\text { HPV-6/11 } \\
/ 16 / 18\end{array}$ & $77(40.3)$ & $73(48.7)$ & $4(9.8)$ & $<0.001$ & $9(4.5)$ & $9(5.8)$ & $0(0.0)$ & 0.098 & $6(3.2)$ & $5(3.5)$ & $1(2.2)$ & 0.681 \\
\hline $\begin{array}{l}\text { HPV-31 } \\
133 / 45\end{array}$ & $50(26.2)$ & $48(32.0)$ & $2(4.9)$ & $<0.001$ & $3(1.5)$ & $3(1.9)$ & $0(0.0)$ & 0.347 & $5(2.6)$ & $2(1.4)$ & $3(6.7)$ & 0.053 \\
\hline $\begin{array}{l}\text { HPV-6/11/16 } \\
/ 18 / 31 / 33 / 45\end{array}$ & $98(51.3)$ & $94(62.7)$ & $4(9.8)$ & $<0.001$ & $11(5.5)$ & $11(7.1)$ & $0(0.0)$ & 0.066 & $10(5.3)$ & $6(4.1)$ & $4(8.9)$ & 0.212 \\
\hline
\end{tabular}




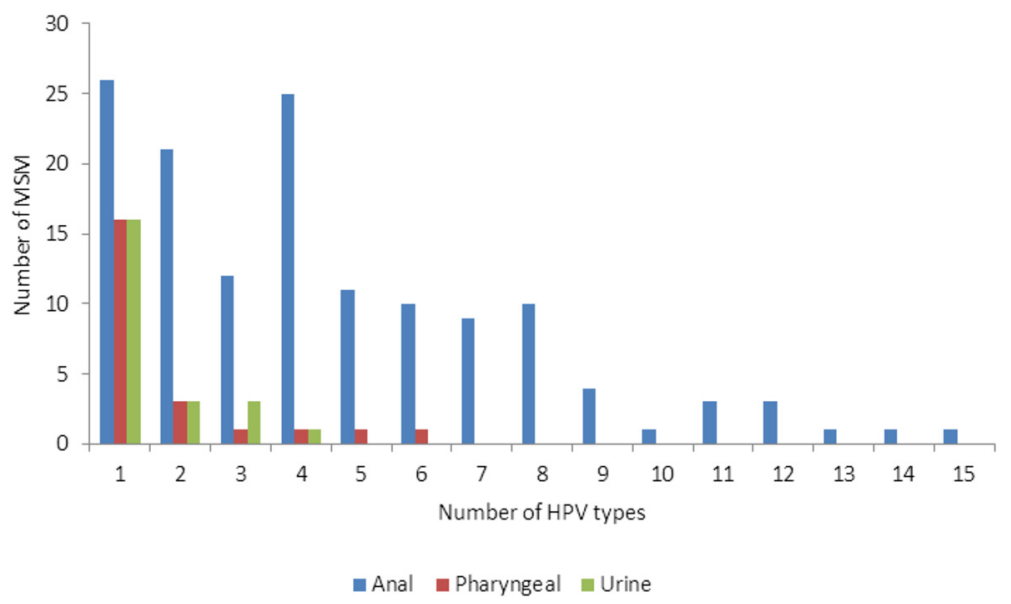

Fig. 1 Number of HPV types in anal, oro-pharyngeal and urine specimens obtained from MSM participants in Cape Town

anal HPV (85.3 \% vs.26.8 \%; $p<0.001)$, multiple anal HPV (70.7 \% vs. $17.1 \% ; p<0.001)$, anal LR-HPV $(78.0 \%$ vs. $22.0 \% ; p<0.001)$, anal HR-HPV $(68.0 \%$ vs. $19.5 \% ; p<0.001)$, anal HPV-6/11/16/18 (48.7 \% vs. $9.8 \% ; p<0.001)$ and anal HPV-6/11/16/18/31/33/45 (62.7\% vs. $9.8 \% ; p<0.001)$. Individuals who had sex with men only were more likely to have any oropharyngeal HPV infection (14.2\% vs. $2.2 \%$; $p=0.027)$ and to have an oro-pharyngeal HR-HPV infection $(9.7 \%$ vs $0.0 \% ; p=0.030)$. No significant differences were observed between MSM only and MSMW with regards to the prevalence of HPV in urine specimens.

\section{Risk factors associated with HPV infections}

Univariate and multiple logistic regression analyses were performed to assess potential and independent associations between participant characteristics/sexual risk factors and HPV infection in this population (Table 4). In univariate analysis having any anal HPV infection was significantly associated with receiving an income $(p=0.044)$, having sex with men only $(p<0.001)$, engaging in group sex in their lifetime $(p=0.002)$, being HIV positive $(p<0.001)$ and practising receptive anal sex $(p<0.001)$. Having a HR-HPV infection in the anal canal was significantly associated with living outside the city $(p=0.050)$, transgender identity $(p=0.031)$, having sex with men only $(p<0.001)$, engaging in group sex in their lifetime $(p=0.006)$, sharing sex toys in last year $(p=0.042)$, being HIV positive $(p<0.001)$, practising receptive anal sex $(p<0.001)$ and having a gonococcal co-infection $(p=0.043)$. Multiple anal HPV infections were detected more frequently in participants that identified as transgender $(p=0.022)$, having sex with men only $(p<0.001)$, being HIV positive $(p<0.001)$, practising receptive anal sex $(p<0.001)$ and having a gonococcal co-infection $(p=0.028)$. Compared to other ethnicities, coloured MSM had significantly less anal HPV infections overall $(p=0.023)$, including HR-HPV $(p=0.003)$ and multiple HPV types $(p=0.013)$. Individuals who participated in transactional sex had fewer multiple anal HPV infections $(p=0.010)$. In multiple logistic regression analyses, having sex with men only (AOR: 10.9, 95 \% CI:

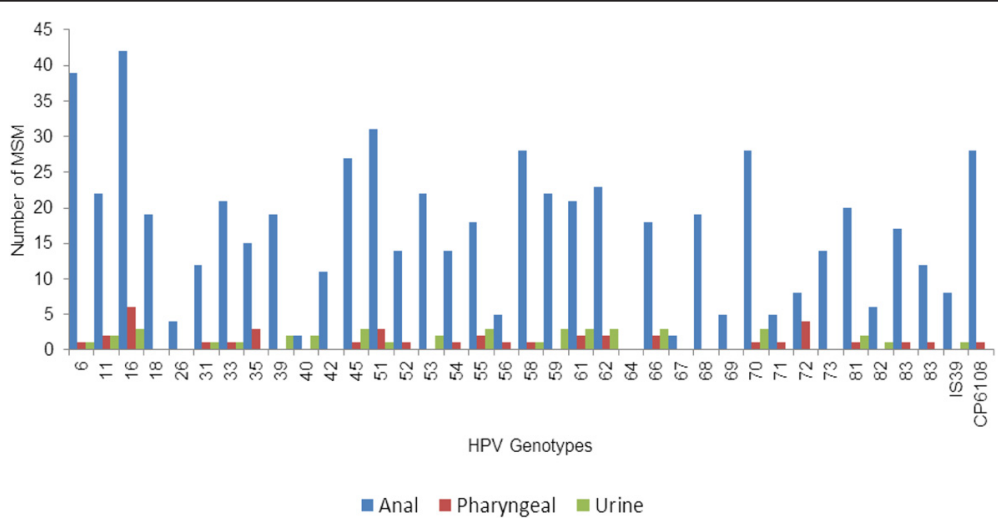

Fig. 2 HPV type-specific prevalence rates in anal, oro-pharyngeal and urine specimens obtained from MSM participants in Cape Town 
Table 4 Univariate and multivariate logistic regression analyses for factors associated with any-, high-risk and multiple anal HPV infections

\begin{tabular}{|c|c|c|c|c|c|c|c|c|c|c|c|c|c|c|c|}
\hline \multirow[t]{2}{*}{ Risk factor } & \multicolumn{5}{|l|}{ Any HPV } & \multicolumn{5}{|l|}{ HR-HPV } & \multicolumn{5}{|c|}{ Multiple HPV } \\
\hline & No (\%) & $\begin{array}{l}\text { COR } \\
(95 \% \mathrm{Cl})\end{array}$ & $p$ & $\begin{array}{l}\text { AOR } \\
(95 \% \mathrm{Cl})\end{array}$ & $p$ & No (\%) & $\begin{array}{l}\text { COR } \\
(95 \% \mathrm{Cl})\end{array}$ & $p$ & $\begin{array}{l}\text { AOR } \\
(95 \% \mathrm{Cl})\end{array}$ & $p$ & No (\%) & $\begin{array}{l}\text { COR } \\
(95 \% \mathrm{Cl})\end{array}$ & $p$ & $\begin{array}{l}\text { AOR } \\
(95 \% \mathrm{Cl})\end{array}$ & $p$ \\
\hline \multicolumn{16}{|l|}{ Gender } \\
\hline Male & $124(89.2)$ & Ref & & & & $97(88.2)$ & Ref & & & & $99(87.6)$ & Ref & & & \\
\hline $\begin{array}{l}\text { Transgender } \\
\text { (MTF) }\end{array}$ & $15(10.8)$ & 1.0 & & & & $13(11.8)$ & $\begin{array}{l}5.3 \\
(1.16-24.16)\end{array}$ & 0.031 & & & $14(12.4)$ & $\begin{array}{l}10.9 \\
(1.40-84.63)\end{array}$ & 0.022 & & \\
\hline \multicolumn{16}{|l|}{ Ethnicity } \\
\hline Black & $46(33.1)$ & Ref & & Ref & & $40(36.4)$ & Ref & & Ref & & 39 (34.5) & Ref & & & \\
\hline Coloured & $35(25.2)$ & $\begin{array}{l}0.4 \\
(0.18-0.88)\end{array}$ & 0.023 & $0.5(0.15-1.42)$ & 0.177 & $24(21.8)$ & $\begin{array}{l}0.3 \\
(0.15-0.67)\end{array}$ & 0.003 & $\begin{array}{l}0.4 \\
(0.13-0.96)\end{array}$ & 0.042 & $26(23.0)$ & $\begin{array}{l}0.4 \\
(0.19-0.82)\end{array}$ & 0.013 & & \\
\hline White & 55 (39.6) & $\begin{array}{l}1.2 \\
(0.50-2.83)\end{array}$ & 0.685 & $0.5(0.17-1.74)$ & 0.303 & $45(40.9)$ & $\begin{array}{l}0.9 \\
(0.44-1.95)\end{array}$ & 0.847 & $\begin{array}{l}0.5 \\
(0.19-1.46)\end{array}$ & 0.219 & 47 (41.6) & $\begin{array}{l}1.2 \\
(0.54-2.42)\end{array}$ & 0.717 & & \\
\hline Other & $3(2.2)$ & $\begin{array}{l}0.9 \\
(0.08-8.85)\end{array}$ & 0.890 & $0.2(0.01-2.14)$ & 0.162 & $1(0.9)$ & $\begin{array}{l}0.2 \\
(0.02-1.62)\end{array}$ & 0.121 & $\begin{array}{l}0.0 \\
(0.00-0.55)\end{array}$ & 0.016 & $1(0.9)$ & $\begin{array}{l}0.2 \\
(0.02-1.75)\end{array}$ & 0.137 & & \\
\hline \multicolumn{16}{|l|}{ Residence } \\
\hline City/suburbs & $96(69.1)$ & Ref & & & & $73(66.4)$ & Ref & & & & $76(67.3)$ & Ref & & & \\
\hline $\begin{array}{l}\text { Informal } \\
\text { settlement }\end{array}$ & $33(23.7)$ & $1.6(0.72-3.73)$ & 0.236 & & & $28(25.5)$ & $\begin{array}{l}1.8 \\
(0.88-3.73)\end{array}$ & 0.108 & & & $28(24.8)$ & $\begin{array}{l}1.7 \\
(0.80-3.42)\end{array}$ & 0.171 & & \\
\hline Outside city & $10(7.2)$ & 1.0 & & & & $9(8.2)$ & $\begin{array}{l}8.1 \\
(1.00-65.96)\end{array}$ & 0.050 & & & $9(8.0)$ & $\begin{array}{l}7.5(0.92- \\
60.49)\end{array}$ & 0.060 & & \\
\hline \multicolumn{16}{|l|}{ Income status } \\
\hline No income & $24(17.3)$ & Ref & & & & $18(16.4)$ & Ref & & & & $20(17.7)$ & Ref & & & \\
\hline Income & $115(82.7)$ & $\begin{array}{l}2.1 \\
(1.02-4.44)\end{array}$ & 0.044 & & & 92 (83.6) & $\begin{array}{l}1.9 \\
(0.94-3.85)\end{array}$ & 0.072 & & & $93(82.3)$ & $\begin{array}{l}1.6 \\
(0.80-3.23)\end{array}$ & 0.187 & & \\
\hline \multicolumn{16}{|c|}{ Sexual behaviour } \\
\hline \multicolumn{16}{|l|}{ Sex partners } \\
\hline $\begin{array}{l}\text { Men and } \\
\text { women }\end{array}$ & $11(2.9)$ & Ref & & Ref & & $8(7.3)$ & Ref & & Ref & & $7(6.2)$ & Ref & & Ref & \\
\hline Men only & $128(92.1)$ & $\begin{array}{l}15.9 \\
(6.95-36.24)\end{array}$ & $<0.001$ & $10.9(3.88-30.81)$ & $<0.001$ & $102(92.7)$ & $\begin{array}{l}8.8 \\
(3.75-20.41)\end{array}$ & $<0.001$ & $\begin{array}{l}4.6 \\
(1.63-12.74)\end{array}$ & 0.005 & $106(93.8)$ & $\begin{array}{l}11.7 \\
(4.82-28.39)\end{array}$ & $<0.001$ & $\begin{array}{l}5.5 \\
(1.92-15.51)\end{array}$ & 0.001 \\
\hline \multicolumn{16}{|c|}{ Engaged in group sex in lifetime } \\
\hline No & $40(28.8)$ & Ref & & Ref & & $30(27.3)$ & Ref & & Ref & & $34(30.1)$ & Ref & & & \\
\hline
\end{tabular}


Table 4 Univariate and multivariate logistic regression analyses for factors associated with any-, high-risk and multiple anal HPV infections (Continued)

Shared sex toys in past 12 months

No $111(79.9)$ Re

Yes $28(20.1) \quad 1.9075-10$

Transactional sex

No $\quad 92(66.2)$ Ref

Paid and/or $\quad 47(33.8) \quad 0.6$

received money

HIV serostatus

$\begin{array}{lll}\text { Negative } & 61(43.9) & \text { Ref } \\ \text { Positive } & 78(56.1) & 8.2 \\ & & (3.46-19.50)\end{array}$

Receptive anal intercourse with men

Never practised 24 (17.3) Ref

$\begin{array}{lll}\text { Practised } & 115(82.7) \quad 7.7\end{array}$

Anal gonorrhoea

Negative

125 (89.9) Ref

Positive

$14(10.1) \quad 1.0$

\section{5 (77.3) Ref}

$25(22.7) \quad 2.4$

$(1.03-5.36)$

0.042

74 (67.3) Ref

$36(32.7) \quad 0.6$

$(0.34-1.10)$

0.100

Ref

$<0.001 \quad 4.1(1.52-11.22) \quad 0.006$

41 (37.3) Ref

$69(62.7) \quad 6.8$

(3.50-13.36)

Ref
$<0.001 \quad 4.0$

(1.82-8.65)

14 (12.7) Ref

Ref

$<0.001 \quad 3.3(1.36-8.09)$

0.009
96 (87.3) $\quad 11.0$

11.0
$(4.97-24.21)$

$<0.001 \quad 4.3$

4.3
$(1.80-10.13)$

98 (89.1) Ref

$12(10.9) \quad 4.8$

$(1.05-22.25)$
88 (77.9) Ref

25 (22.1) 2.2

(0.95-4.97)

0.064

79 (69.9) Ref

$34(30.1) \quad 0.5$

(0.25-0.82)

0.010

40 (35.4) Ref

Ref

$<0.001 \quad 6.9$

(4.86-20.74)

(3.10-15.42)

$<0.001$

14 (12.4) Ref

Ref

99 (87.6) $\quad 11.3$

11.3
$(5.13-24.95)$

$<0.001 \quad 4.2$

(1.79-9.65)

0.001
100 (88.5) Ref

$13(11.5) \quad 10.0$

$(1.28-78.19)$ 
$3.88-30.81, p<0.001)$, engaging in group sex in lifetime (AOR: 4.7, 95 \% CI: 1.79-12.43, $p=0.002$ ), being HIVpositive (AOR: 4.1, $95 \% \mathrm{CI}: 1.52-11.22, p=0.006$ ) and practising receptive anal intercourse (AOR: 3.3, $95 \% \mathrm{CI}$ : $1.36-8.09, p=0.009$ ) were the only factors associated with having any anal HPV infection after adjusting for confounders and other variables found significant in the univariate analysis. Coloured ethnicity was associated with significantly less anal HR-HPV infections (AOR: 0.4, $95 \%$ CI: $0.13-0.96, p=0.042$ ) while having sex with men only (AOR: 4.6, 95 \% CI: 1.63-12.74, $p=0.005$ ), engaging in group sex in lifetime (AOR: 3.1, $95 \%$ CI: 1.357.07, $p=0.006$ ), being HIV-positive (AOR: 4.1, $95 \%$ CI: $1.89-8.83, p<0.001)$ and practicing receptive anal intercourse (AOR: 4.3, 95 \% CI: 1.80-10.13, $p<0.001$ ) were all associated with having a greater likelihood of infection by a HR-HPV type. Having multiple anal HPV infections were independently associated with having sex with men only (AOR: 5.5, 95 \% CI: 1.9215.51, $p=0.001$ ), being HIV-positive (AOR: 6.9, $95 \%$ CI: $3.10-15.42, p<0.001)$ and practising receptive anal intercourse (AOR: 4.2, 95 \% CI: 1.79-9.65, $p=0.001$ ). Age group, education, job status, partner status, number of male sexual partners in the past 12 months, sex at sex-onsite venues, sex with a partner met through the internet, engaging in group sex in the past 12 months, alcohol and drug use during sex, having had a STI in the past 12 months, syphilis serostatus and having an anal chlamydial infection were not significantly associated with the three HPV outcomes in the anal canal. Further, no risk factors were independently associated with oro-pharyngeal HPV infections or with HPV detected in the urine.

\section{Discussion}

This study reports HPV prevalence and risk factors associated with HPV infections at three different anatomical sites among high-risk MSM in Cape Town. We found a high prevalence of HR-HPV (57.6\%) in the anal canal of study participants. HPV genotypes were less frequently detected in the pharynx $(11.5 \%)$ and urine $(15.3 \%)$ of study participants. Previous studies have shown that anal HPV infection is more common among MSM than heterosexual men (MSW), and that anal HPV prevalence rates can be 4-10 times higher in MSM compared to MSW $[19,20]$. The high prevalence of anal HPV infection observed in this study is similar to anal HPV prevalence rates for high-risk MSM reported in Peru, Italy and Thailand but higher than anal HPV prevalence rates reported in countries such as Australia and Puerto Rico [9, 21-24].

MSM who are HIV-positive have an increased risk of anal HPV infection and anal cancer. HPV prevalence rates of 89-93 \% have been reported among HIV- positive MSM in a recent systematic review and metaanalysis of 53 studies [7]. The incidence of anal cancer among HIV-positive MSM is more than 80-fold higher than that observed in the general population [25]. HIV infection has been shown to increase susceptibility to persistent HPV, increasing the risk of acquiring new $\mathrm{HPV}$ infections and reactivation of latent HPV infections [12]. Persons who are at high risk for HIV acquisition may be at higher risk of HPV infection due to the same high risk sexual practises [11]. As shown in Table 2, HIV-positive MSM had significantly more anal HPV infections overall (91.8 \% HIV-infected vs. $57.6 \%$ HIVuninfected), including LR-HPV, HR-HPV, multiple HPV types and HPV vaccine types (HPV-6/11/16/18 + 31/33/45). The most common LR-HPV and HR-HPV types detected in the anal specimens of all MSM as well as HIV-positive MSM were HPV-6 and HPV-16 respectively, followed by high risk types HPV-58, HPV-45 and HPV-51. HPV16 was also the most common HR-HPV type found in HIV-positive MSM from Thailand, USA, China, Spain and Canada [9, 12, 26-28].

Multiple anal HPV infections are commonly described among MSM, especially HIV-positive MSM [8, 26-28]. Having more than one HPV type detected was more common in the anal canal of MSM while single HPV infections were more frequently detected in the pharynx and urine. In this study, multiple HPV types were detected in the anal specimens of $59.2 \%$ of all MSM, $81.3 \%$ of all HPV-positive MSM and $85.9 \%$ of HIVpositive MSM. Among an HIV-positive MSM cohort in Canada (HIPVIRG cohort study), the majority of participants $(90.9 \%)$ were infected with multiple HPV types in the anal canal, ranging between 0 and $18 \mathrm{HPV}$ types (mean 5 HPV types) [12]. More recently, Supindham et al. [9] reported a $100 \% \mathrm{HPV}$ co-infection rate in the anal specimens of HIV-positive MSM in Thailand, with the majority being multiple HPV infections (mean 4.8 HPV types). The mean number of anal HPV types detected in HPV-positive MSM in this study was 4.5 types, which increased to 5.7 types among HIV-positive MSM $(p<0.001)$. The highest number of HPV types detected in a single anal sample was $15 \mathrm{HPV}$ types in an HIV-positive participant co-infected with anal gonorrhoea and chlamydia. The mean number of HPV types in the oropharynx and urine was 1.7 and 1.4 types respectively, and the number of HPV types at these anatomical sites did not differ significantly according to HIV status. Multiple anal HPV infections are a cause for concern due to the strong association with anal intraepithelial neoplasia (AIN) and the development to high-grade AIN and anal cancer [29, 30].

Oro-pharyngeal HPV infections are much less common in adults than anogenital HPV infections [31]. King et al. [14] reported that the majority of HPV infections detected in the oral cavity of MSM in London were single HPV 
types. However, the incidence of HPV-related oropharyngeal cancers in the general population is increasing relative to other oral cancers associated with alcohol and tobacco use. A number of HR-HPV types have been implicated in carcinogenesis and tumour progression at different anatomical sites and are mainly due to the actions of their E6 and E7 viral oncogenes, which induce and maintain cellular transformation [32]. The majority of oropharyngeal squamous cell carcinomas (OPSCC) (+ - $90 \%$ of cases) are associated with HPV-16 infection; however other HR-HPV types have also been identified in HPVpositive OPSCC and these types, although rare, constitute an important subset of oro-pharyngeal cancers which should not be ignored [33]. A few studies have reported associations between non-HPV-16 high-risk types and oro-pharyngeal cancer after controlling for or excluding HPV-16 infection [34-36]. A large US case-control study demonstrated that combined infections with HPV-16 and other HR-HPV types significantly increased the risk of head and neck squamous cell carcinoma [36]. Further studies are needed to determine whether oro-pharyngeal tumours containing non-HPV-16 high-risk types present less often with lymph node metastases and have better treatment outcomes [33, 37]. Davidson et al [38] reported a HPV prevalence of $5.6 \%$ in oral rinse and gargle samples among male factory workers in Pretoria, South Africa, aged 17 - 64 years. Although oral sex was not common in this population, it was practised among men identified with HR-HPV infection [38]. Performing oral and oroanal sex on men and women were not associated with oro-pharyngeal HPV infections in this study. However, HIV-positive MSM had significantly more oro-pharyngeal HPV infections (17.1 \% in HIV-positive vs. $7.1 \%$ in HIVnegative; $p=0.029)$, including LR-HPV infections $(11.4 \%$ in HIV-positive vs. $3.6 \%$ in HIV-negative; $p=0.032)$. It is not clear if the increased oral HPV incidence in HIVpositive individuals is due to HIV-related immunosuppression or due to risky sexual practises [39]. In Italy, Parisi et al. [40] detected HPV in the oral specimens of approximately $20 \%$ of HIV-infected MSM, which is lower than that reported by Blas et al. [41] (44.3\%) in Peru, but higher than the oro-pharyngeal HPV prevalence rates in HIV-positive MSM reported in this study. In our study, having any HPV infection or a LR-HPV infection in the oropharynx were the only oro-pharyngeal HPV groupings associated with being HIV-positive. No differences were observed between HIV-negative and HIV-positive MSM with regards to the prevalence of single HPV types, multiple HPV types, HR-HPV or HPV vaccine types detected in the oropharynx.

This study showed that MSM only had over three times more anal HPV infections and over 6 times more oro-pharyngeal HPV infections than MSMW. Anal HPV prevalence rates among MSM only were significantly higher for all HPV groupings, except for single HPV type prevalence. Having any oro-pharyngeal HPV infection or an oro-pharyngeal HR-HPV infection were the only oropharyngeal HPV groupings that showed significant differences between MSM only and MSMW. No significant differences were observed in the prevalence of HPV in urine between MSM only and MSMW. Compared to other male populations higher anal HPV prevalence rates are frequently reported for MSM only [19, 24, 42]. Limited information is available in the literature describing HPV prevalence rates in MSMW, especially anal HPV prevalence rates. Lu et al. [43] studied the seroprevalence of quadrivalent HPV vaccine types (HPV$6,11,16,18)$ among men with different sexual practises in Brazil, Mexico and USA (The HIM study) and found MSMW to have lower HPV seroprevalence rates than MSM but higher than in MSW. Genital HPV prevalence analysis in the same HIM study cohort showed that MSMW had higher HPV prevalence rates on the penis and scrotum compared to MSM and MSW, which could be attributed to increased number of sex partners and increased frequency of insertive sex acts among MSMW. In our study, in comparison with the MSM only group, significantly fewer MSMW were HIV-positive, practised receptive anal intercourse with men whilst more MSMW practised insertive anal intercourse with men. These sexual practises could account for the absence of gonococcal and chlamydial co-infections detected in the anal canal of MSMW and the increased number of chlamydial coinfections detected in the urine of MSMW. MSMW were also less likely to be syphilis seropositive. Even though anal HPV infection among MSMW who practise receptive anal intercourse could be linked to these sexual practises, anal HPV transmission can also be attributed to other factors, similar to those found among HPV-infected MSW, such as transmission through skin contact, sharing sex toys or vertical transmission. Genital HPV genotypes have been detected on fingers and HPV transmission between anogenital sites and hands have previously been reported among heterosexual couples [44]. These alternative modes of HPV transmission could explain the observation that $17.3 \%$ of men who indicated no history of receptive anal intercourse had HPV detected in the anal canal.

This study had a number of limitations. Firstly, the relatively small sample size may have resulted in the misidentification of some predictors of HPV infection, especially in oro-pharyngeal and urine samples where the prevalence of HPV was low. Secondly, the liquid oral rinse sampling method has been shown to have a higher sensitivity than oro-pharyngeal swabs for the detection of HPV in the mouth and throat and therefore the use of oro-pharyngeal swabs in this study could have decreased the HPV detection rate in the oropharynx [13]. Thirdly, we were only able to test DNA extracts from 
urine as an indicator of urethral HPV infection. This was due to the sampling undertaken in the primary study; the true prevalence of penile HPV may have been higher than that reported here if penile samples had also been available for additional HPV testing. Fourthly, the participants attended one clinic in Cape Town and the findings of this study might not be generalized to the South African MSM population.

In keeping with South Africa's National Strategic Plan for HIV and AIDS, STIs and TB, scaling up services for most at risk populations (including MSM) to reduce HIV/STI burden should be prioritised. For HPV, these efforts include targeted behavioural interventions such as promotion of proper and consistent condom use, medical male circumcision to reduce the incidence, prevalence and persistence of HPV infections in MSM and HPV vaccination of boys. The South African government is currently providing free HPV vaccinations for 9-10 year old girls in all public schools in South Africa but it is unlikely that MSM would benefit from herd immunity due to female vaccination roll-out and it would be optimal to vaccinate boys directly. Randomised controlled trials have shown HPV vaccination to be highly efficacious in MSM with the potential to substantially reduce the burden of HPV-associated disease in this population [45]. More studies are needed to determine the natural history and burden of HPV-associated diseases in South African MSM in order to formulate effective HPV prevention strategies for the future.

\section{Conclusions}

A high prevalence of anal HPV infection was observed in this MSM population in Cape Town. All HPV groupings (any HPV, LR-HPV, HR-HPV, single type, multiple type, quadrivalent vaccine types) were more prevalent in the anal canal of HIV-positive MSM. Risk factors associated with any HPV, HR-HPV and multiple HPV types in the anal canal included having sex with men only, being HIV-positive and practising receptive anal intercourse. No risk factors were independently associated with oropharyngeal HPV infection or HPV detected in urine. Targeted interventions and HPV vaccination of boys should be considered to reduce the burden of HPVrelated diseases among MSM in South Africa.

\footnotetext{
Abbreviations

AOR, adjusted odds ratio; $\mathrm{Cl}$, confidence interval; COR, crude odds ratio; HIV, human immunodeficiency virus; HPV, human papillomavirus; HR, high-risk; IQR, interquartile range; ISH, in situ hybridisation; ITCMH, Ivan Toms Centre for Men's Health; LA, linear Array; LR, low-risk; MSM, men-who-have-sex-withmen; MSMW, men-who-have-sex-with-men-and-women; MSW, men-whohave-sex-with-women; NICD, National Institute for Communicable Diseases; SD, standard deviation; SPM, sexual partner matrix; STI, sexually transmitted infection; TGW, transgender women
}

\section{Acknowledgments}

The authors would like to thank staff that collected specimens and questionnaire data at the Ivan Toms Centre for Men's Health in Woodstock, Cape Town and study participants for their cooperation in this study. Special thanks go to Dr Tendesayi Kufa-Chakezha for her assistance with statistical analysis and general comments related to the manuscript.

\section{Funding}

The sub study was funded by the Global Disease Detection (GDD) programme grant no. 1U19GH000622-01 while the main study received funding and support from PEPFAR/USAID and the Anova Health Institute NPC. The Anova Health Institute NPC is supported by the US President's Emergency Plan for AIDS Relief (PEPFAR) program via the US Agency for International Development, (USAID) under Cooperative Agreement No. AID-674-A-12-00015.

\section{Availability of data and materials}

Individual specimens and questionnaire data were delinked from all personal identifiers and each participant was assigned with a unique random HPV study number to ensure anonymity. There were no documents in existence linking original study numbers with HPV study numbers. The HPV genotyping results obtained in this sub study were therefore not relayed to any patients and the results were only used for research purposes. Delinked data are stored at the STI Section, Centre for HIV and STIS, NICD and can be obtained upon request from the corresponding author.

\section{Authors' contributions}

EEM worked on the conceptualization of the sub study, study design, ethics approval, laboratory testing, data analysis, interpretation of data and manuscript preparation; KR worked on study design, ethics approval, specimen and data collection, interpretation of data and manuscript preparation; TFC worked on data analysis and interpretation of data; HS and JM worked on the conceptualization of the original study and manuscript preparation; DAL worked on the conceptualization of the study, interpretation of data and manuscript preparation. All authors read and approved the final manuscript.

\section{Competing interests}

The authors declare that they have no competing interests.

\section{Consent for publication}

Not applicable.

\section{Ethics approval and consent to participate}

All 200 participants, 18 years of age or older, provided informed consent and gave permission for their specimens to be used for future research. Ethical approvals for this study were obtained from the Human Research Ethics Committee (Medical) of the University of the Witwatersrand [protocol M120454] and the Bioethics Department at the University of Cape Town [protocol 419/2011].

\section{Author details}

${ }^{1}$ Centre for HIV and Sexually Transmitted Infections, National Institute for Communicable Diseases, National Health Laboratory Service, Johannesburg, South Africa. ${ }^{2}$ Anova Health Institute, Johannesburg, South Africa. ${ }^{3}$ Anova Health Institute, Cape Town, South Africa. ${ }^{4}$ Department of Medicine, Division of Infectious Diseases and HIV Medicine, University of Cape Town, Cape Town, South Africa. ${ }^{5}$ Division of Epidemiology and Biostatistics, School of Public Health, University of the Witwatersrand, Johannesburg, South Africa. ${ }^{6}$ Division of Epidemiology \& Biostatistics, School of Public \& Family Medicine, University of Cape Town, Cape Town, South Africa. ${ }^{7}$ Western Sydney Sexual Health Centre, Western Sydney Local Health District, Parramatta, Australia.

${ }^{8}$ Marie Bashir Institute for Infectious Diseases and Biosecurity \& Sydney Medical School-Westmead, University of Sydney, Sydney, Australia.

Received: 20 April 2016 Accepted: 12 July 2016

Published online: 22 August 2016

\section{References}

1. Giuliano AR, Tortolero-Luna G, Ferrer E, Burchell AN, de Sanjose S, Kjaer SK, Munoz N, Schiffman M, Bosch FX. Epidemiology of human papillomavirus infection in men, cancers other than cervical and benign conditions. vaccine. 2008;26 Suppl 10:K17-28. 
2. Rositch AF, Mao L, Hudgens MG, Moses S, Agot K, Backes DM, Nyagaya E, Snijders PJ, Meijer CJ, Bailey RC, et al. Risk of HIV acquisition among circumcised and uncircumcised young men with penile human papillomavirus infection. AIDS. 2014;28(5):745-52.

3. Mbulawa ZZ, Marais DJ, Johnson LF, Coetzee D, Williamson AL. Impact of human immunodeficiency virus on the natural history of human papillomavirus genital infection in South African men and women. J Infect Dis. 2012;206(1):15-27

4. Shisana O, Rehle T, Simbayi LC, Zuma K, Jooste S, Zungu N, Labadarios D, Onoya D. South African National HIV Prevalence, Incidence and Behaviour Survey. Cape Town: HSRC Press; 2012. ISBN 978-0-7969-2456-8 2014.

5. Human Sciences Research Council. Three cities study: high levels of HIV infection found among men who have sex with men: The South African Marang Men's Project. 2014. http://www.hsrc.ac.za/en/media-briefs/hiv-aidsstis-and-tb/marang. Accessed 13 Aug 2015.

6. Baggaley RF, White RG, Boily MC. HIV transmission risk through anal intercourse: systematic review, meta-analysis and implications for HIV prevention. Int J Epidemiol. 2010;39(4):1048-63.

7. Machalek DA, Poynten M, Jin F, Fairley CK, Farnsworth A, Garland SM, Hillman RJ, Petoumenos K, Roberts J, Tabrizi SN, et al. Anal human papillomavirus infection and associated neoplastic lesions in men who have sex with men: a systematic review and meta-analysis. Lancet Oncol. 2012;13(5):487-500.

8. Mendez-Martinez R, Rivera-Martinez NE, Crabtree-Ramirez B, Sierra-Madero JG, Caro-Vega Y, Galvan SC, de Leon DC, Garcia-Carranca A. Multiple human papillomavirus infections are highly prevalent in the anal canal of human immunodeficiency virus-positive men who have sex with men. BMC Infect Dis. 2014;14:671.

9. Supindham $T$, Chariyalertsak $S$, Utaipat U, Miura T, Ruanpeng D, Chotirosniramit N, Kosashunhanan N, Sugandhavesa P, Saokhieo P, Songsupa R, et al. High Prevalence and Genotype Diversity of Anal HPV Infection among MSM in Northern Thailand. PLoS One. 2015;10(5):e0124499.

10. Wirtz AL, Zelaya CE, Peryshkina A, McGowan I, Cranston RD, Latkin C, Galai N, Mogilniy V, Dzhigun P, Kostetskaya I, et al. Anal human papillomavirus and HIV: A cross-sectional study among men who have sex with men in Moscow, Russia, 2012-2013. Euro Surveill. 2015;20(15):pii=21095.

11. Hu Y, Oian HZ, Sun J, Gao L, Yin L, Li X, Xiao D, Li D, Sun X, Ruan Y, et al. Anal human papillomavirus infection among HIV-infected and uninfected men who have sex with men in Beijing, China. J Acquir Immune Defic Syndr. 2013;64(1):103-14

12. de Pokomandy A, Rouleau D, Ghattas G, Vezina S, Cote P, Macleod J, Allaire G, Franco EL, Coutlee F. Prevalence, clearance, and incidence of anal human papillomavirus infection in HIV-infected men: the HIPVIRG cohort study. J Infect Dis. 2009:199(7):965-73.

13. Read TR, Hocking JS, Vodstrcil LA, Tabrizi SN, McCullough MJ, Grulich AE, Garland SM, Bradshaw CS, Chen MY, Fairley CK. Oral human papillomavirus in men having sex with men: risk-factors and sampling. PLoS One. 2012; 7(11):e49324.

14. King EM, Gilson R, Beddows S, Soldan K, Panwar K, Young C, Jit M, Edmunds WJ, Sonnenberg P. Oral human papillomavirus (HPV) infection in men who have sex with men: prevalence and lack of anogenital concordance. Sex Transm Infect. 2015;91(4):284-6.

15. Shigehara K, Sasagawa T, Namiki M. Human papillomavirus infection and pathogenesis in urothelial cells: a mini-review. J Infect Chemother. 2014; 20(12):741-7.

16. SANAC. National Strategic Plan on HIV, STIs and TB: 2012-2016. Edited by SANAC. Pretoria: SANAC; 2012.

17. Rebe K, Lewis D, Myer L, de Swardt G, Struthers H, Kamkuemah M, McIntyre J. A Cross Sectional Analysis of Gonococcal and Chlamydial Infections among Men-Who-Have-Sex-with-Men in Cape Town, South Africa. PLoS One. 2015;10(9):e0138315.

18. South African Department of Health. First Line Comprehensive Management and Control of Sexually Transmitted Infections (STIS). Protocol for the management of a person with a sexually transmitted infection according to the essential drug list. 2003. Department of Health. http://www.nicd.ac.za/assets/files/STI\%20Book\%202008\% 20Edited(1).pdf. Accessed 07 June 2016

19. Nyitray AG, da Silva RJ C, Baggio ML, Lu B, Smith D, Abrahamsen M, Papenfuss M, Villa LL, Lazcano-Ponce E, Giuliano AR. Age-specific prevalence of and risk factors for anal human papillomavirus (HPV) among men who have sex with women and men who have sex with men: the HPV in men (HIM) study. J Infect Dis. 2011;203(1):49-57.
20. Goldstone S, Palefsky JM, Giuliano AR, Moreira Jr ED, Aranda C, Jessen H, Hillman RJ, Ferris DG, Coutlee F, Liaw KL, et al. Prevalence of and risk factors for human papillomavirus (HPV) infection among HIV-seronegative men who have sex with men. J Infect Dis. 2011;203(1):66-74.

21. Quinn R, Salvatierra J, Solari V, Calderon M, Ton TG, Zunt JR. Human papillomavirus infection in men who have sex with men in Lima, Peru. AIDS Res Hum Retroviruses. 2012;28(12):1734-8.

22. Latini A, Dona MG, Ronchetti L, Giglio A, Moretto D, Colafigli M, Laquintana V, Frasca M, Zaccarelli M, Antinori A, et al. Prevalence of anal human papillomavirus infection and cytologic abnormalities among HIV-infected and HIV-uninfected men who have sex with men. J Int AIDS Soc. 2014;17(4 Suppl 3):19662.

23. Zou H, Tabrizi SN, Grulich AE, Garland SM, Hocking JS, Bradshaw CS, Morrow A, Prestage G, Cornall AM, Fairley CK, et al. Early acquisition of anogenital human papillomavirus among teenage men who have sex with men. J Infect Dis. 2014;209(5):642-51.

24. Colon-Lopez V, Ortiz AP, Del Toro-Mejias L, Clatts MC, Palefsky JM Epidemiology of anal HPV infection in high-risk men attending a sexually transmitted infection clinic in Puerto Rico. PLoS One. 2014;9(1):e83209.

25. Silverberg MJ, Lau B, Justice AC, Engels E, Gill MJ, Goedert JJ, Kirk GD, D'Souza G, Bosch RJ, Brooks JT, et al. Risk of anal cancer in HIV-infected and HIVuninfected individuals in North America. Clin Infect Dis. 2012;54(7):1026-34.

26. Gao L, Zhou F, Li X, Yang Y, Ruan Y, Jin Q. Anal HPV infection in HIV-positive men who have sex with men from China. PLoS One. 2010;5(12):e15256.

27. Torres M, Gonzalez C, del Romero J, Viciana P, Ocampo A, Rodriguez-Fortunez P, Masia M, Blanco JR, Portilla J, Rodriguez C, et al. Anal human papillomavirus genotype distribution in HIV-infected men who have sex with men by geographical origin, age, and cytological status in a Spanish cohort. J Clin Microbiol. 2013;51(11):3512-20.

28. Sahasrabuddhe W, Castle PE, Follansbee S, Borgonovo S, Tokugawa D, Schwartz LM, Lorey TS, LaMere BJ, Gage JC, Fetterman B, et al. Human papillomavirus genotype attribution and estimation of preventable fraction of anal intraepithelial neoplasia cases among HIV-infected men who have sex with men. J Infect Dis. 2013;207(3):392-401.

29. Coutlee F, de Pokomandy A, Franco EL. Epidemiology, natural history and risk factors for anal intraepithelial neoplasia. Sex Health. 2012;9(6):547-55.

30. Sendagorta E, Herranz P, Guadalajara H, Zamora FX. [Early detection of anal intraepithelial neoplasia in high-risk patients]. Actas Dermosifiliogr. 2011; 102(10):757-65.

31. D'Souza G, Fakhry C, Sugar EA, Seaberg EC, Weber K, Minkoff HL, Anastos K, Palefsky JM, Gillison ML. Six-month natural history of oral versus cervical human papillomavirus infection. Int J Cancer. 2007;121(1):143-50.

32. Narisawa-Saito M, Kiyono T. Basic mechanisms of high-risk human papillomavirus-induced carcinogenesis: roles of E6 and E7 proteins. Cancer Sci. 2007:98(10):1505-11.

33. Walline HM, Komarck C, MCHugh JB, Byrd SA, Spector ME, Hauff SJ, Graham MP, Bellile E, Moyer JS, Prince ME, et al. High-risk human papillomavirus detection in oropharyngeal, nasopharyngeal, and oral cavity cancers: comparison of multiple methods. JAMA Otolaryngol Head Neck Surg. 2013;139(12):1320-7.

34. Anantharaman D, Gheit T, Waterboer T, Abedi-Ardekani B, Carreira C, McKay-Chopin S, Gaborieau V, Marron M, Lagiou P, Ahrens W, et al. Human papillomavirus infections and upper aero-digestive tract cancers: the ARCAGE study. J Natl Cancer Inst. 2013;105(8):536-45.

35. Ribeiro KB, Levi JE, Pawlita M, Koifman S, Matos E, Eluf-Neto J, Wunsch-Filho V, Curado MP, Shangina O, Zaridze D, et al. Low human papillomavirus prevalence in head and neck cancer: results from two large case-control studies in high-incidence regions. Int J Epidemiol. 2011;40(2):489-502.

36. Michaud DS, Langevin SM, Eliot M, Nelson HH, Pawlita M, McClean MD, Kelsey KT. High-risk HPV types and head and neck cancer. Int J Cancer. 2014;135(7):1653-61.

37. Nichols AC, Dhaliwal SS, Palma DA, Basmaji J, Chapeskie C, Dowthwaite S, Franklin JH, Fung K, Kwan K, Wehrli B, et al. Does HPV type affect outcome in oropharyngeal cancer? J Otolaryngol Head Neck Surg. 2013:42:9.

38. Davidson CL, Richter KL, Van der Linde M, Coetsee J, Boy SC. Prevalence of oral and oropharyngeal human papillomavirus in a sample of South African men: a pilot study. S Afr Med J. 2014;104(5):358-61.

39. Mooij SH, Boot HJ, Speksnijder AG, Meijer CJ, King AJ, Verhagen DW, de Vries HJ, Quint WG, Molijn A, de Koning MN, et al. Six-month incidence and persistence of oral HPV infection in HIV-negative and HIV-infected men who have sex with men. PLoS One. 2014;9(6):e98955. 
40. Parisi SG, Cruciani M, Scaggiante R, Boldrin C, Andreis S, Dal Bello F, Pagni S, Barelli A, Sattin A, Mengoli C, et al. Anal and oral human papillomavirus (HPV) infection in HIV-infected subjects in northern Italy: a longitudinal cohort study among men who have sex with men. BMC Infect Dis. 2011;11:150.

41. Blas MM, Brown B, Menacho L, Alva IE, Silva-Santisteban A, Carcamo C. HPV Prevalence in Multiple Anatomical Sites among Men Who Have Sex with Men in Peru. PLoS One. 2015;10(10):e0139524.

42. Nyitray AG, da Silva RJ C, Baggio ML, Smith D, Abrahamsen M, Papenfuss M, Lin HY, Quiterio M, Salmeron J, Lazcano-Ponce E, et al. Six-month incidence, persistence, and factors associated with persistence of anal human papillomavirus in men: the HPV in men study. J Infect Dis. 2011;204(11):1711-22.

43. Lu B, Viscidi RP, Lee JH, Wu Y, Villa LL, Lazcano-Ponce E, da Silva RJ, Baggio ML, Quiterio M, Salmeron J, et al. Human papillomavirus (HPV) 6, 11, 16, and 18 seroprevalence is associated with sexual practice and age: results from the multinational HPV Infection in Men Study (HIM Study). Cancer Epidemiol Biomarkers Prev. 2011:20(5):990-1002.

44. Hernandez BY, Wilkens LR, Zhu X, Thompson P, McDuffie K, Shvetsov YB, Kamemoto LE, Killeen J, Ning L, Goodman MT. Transmission of human papillomavirus in heterosexual couples. Emerg Infect Dis. 2008;14(6):888-94.

45. Palefsky J, Giuliano A. Quadrivalent HPV vaccine efficacy in men having sex with men. Malmo: Paper presented at: International Papillomavirus Conference; May 2009; 2009.

\section{Submit your next manuscript to BioMed Central and we will help you at every step:}

- We accept pre-submission inquiries

- Our selector tool helps you to find the most relevant journal

- We provide round the clock customer support

- Convenient online submission

- Thorough peer review

- Inclusion in PubMed and all major indexing services

- Maximum visibility for your research

Submit your manuscript at www.biomedcentral.com/submit 